\title{
Entanglement in a class of multiqubit mixed states without multipartite tangles
}

\author{
Yan-Kui Bai, ${ }^{1,2}$ Ming-Yong Ye, ${ }^{1,3}$ and Z. D. Wang1* \\ 1 Department of Physics and Center of Theoretical and Computational Physics, \\ University of Hong Kong, Pokfulam Road, Hong Kong, People's Republic of China \\ ${ }^{2}$ College of Physical Science and Information Engineering \& Hebei Advance Thin Films Laboratory, \\ Hebei Normal University, Shijiazhuang, Hebei 050016, People's Republic of China \\ ${ }^{3}$ School of physics and Optoelectronics Technology, \\ Fujian Normal University, Fuzhou 350007, People's Republic of China
}

\begin{abstract}
Based on quantum complementary relations (QCRs) and a purification scenario, we analyze a class of $\mathrm{N}$-qubit mixed states that are entangled but do not have two-, and genuine three-, four, ..., N-qubit entanglements. It is shown that entanglement (one-tangle or negativity) in these mixed states is closely related to the QCR entanglement of their purified states. In particular, it is elaborated that when the mixed state does not have multipartite tangles (two- and higher tangles), its entanglement is actually a kind of genuine multipartite QCR entanglement between the system and its environment.
\end{abstract}

PACS numbers: 03.67.Mn, 03.65.Ud, 03.65.Ta

Entanglement plays a crucial role in quantuminformation processing, including quantum communication [1, 2, 3] and quantum computation [4, 5, 6]. Therefore, it is highly desirable and necessary to characterize the entanglement property of quantum systems. So far, only bipartite entanglement has been understood well in many aspects 7], while the characterization of multipartite entanglement for quantum many-body systems, especially for their mixed states, has still been very challenging despite a number of profound results, e.g., [8, 9].

Uhlmann worked out that mixed-state entanglement measure may be constructed by the convex roof extension of pure-state measure [10]. For example, when an entanglement measure $E(\psi)$ is available for pure states, the corresponding measure for a given mixed state can be expressed as

$$
E(\rho)=\min \sum p_{i} E\left(\psi_{i}\right)
$$

where the minimum runs over all the pure-state decomposition $\left\{p_{i}, \psi_{i}\right\}$ of $\rho$. As a result, an analytical entanglement formula of any two-qubit system, i.e., the concurrence $C(\rho)$, was derived [11], and has been widely accepted, though the analytical results for generic multiqubit systems can hardly be obtained.

As is known, entanglement of a multi-qubit pure state has likely a hierarchy structure: it is contributed from different levels of quantum correlation. A quantitative relation for a three-qubit pure state is given by Coffman, Kundu, and Wootters (CKW) [12] $\tau_{A}\left(\psi_{A B C}\right)=$ $C^{2}\left(\rho_{A B}\right)+C^{2}\left(\rho_{A C}\right)+\tau_{3}\left(\psi_{A B C}\right)$, where the linear entropy (one-tangle) $\tau_{A}$ [13] quantifies the bipartite entanglement in partition $A \mid B C$, and the square of concurrence $C^{2}$ (two-tangle) and three-tangle $\tau_{3}$ [14] quantify two- and genuine three-qubit entanglement, respectively.

*Electronic address: zwang@hkucc.hku.hk
Recently, Lohmayer et al [15] analyzed the CKW relation for a kind of mixed state $\rho_{A B C}$, in which the bipartite entanglement $\tau_{A}$ is nonzero while no two- and three-qubit entanglement is present. This is remarkably different from that in the pure state cases. More interestingly, we even find below that this feature exists for a class of $N$-qubit $(N>2)$ mixed states, i.e., the mixed state is entangled but all the two-, three-, ... , and Ntangles vanish. Thus it is natural to ask where this kind of entanglement comes from.

A mixed state can be interpreted as the partial trace of a larger pure state composed of the concerned system and its environment [16]. In this sense, by analyzing the pure state, one may obtain more valuable information about the mixed state.

In this paper, motivated by the above question, we analyze in detail the entanglement in this class of $N$-qubit mixed states without multipartite tangles. It is shown that this kind of mixed state entanglement is closely related to their purified states. In particular, it is elaborated that the one-tangle (or negativity [17]) in this class of mixed states is a kind of genuine multipartite QCRentanglement between the system and its environment, focusing on (i) the three-qubit mixed state addressed by Lohmayer et al before; (ii) a new four-qubit case, namely the one-parameter Smolin state [18]; and (iii) a more general $N$-qubit case.

The QCRs and a multipartite entanglement measureAs a fundamental principle, the QCR is often referred to the mutually exclusive properties of a single quantum system. A quantitative version in an N-qubit pure state $\left|\Psi_{N}\right\rangle$ is [19]

$$
\tau_{k\left(R_{k}\right)}+S_{k}^{2}=1,
$$

where the linear entropy $\tau_{k\left(R_{k}\right)}=2\left(1-\operatorname{tr} \rho_{k}^{2}\right)$ [13] characterizes the bipartite quantum entanglement between qubit $k$ and the remaining qubits $R_{k}$, and $S_{k}^{2}$ is a measure of single-particle property. Based on this relation, a multipartite QCR-entanglement measure is introduced 


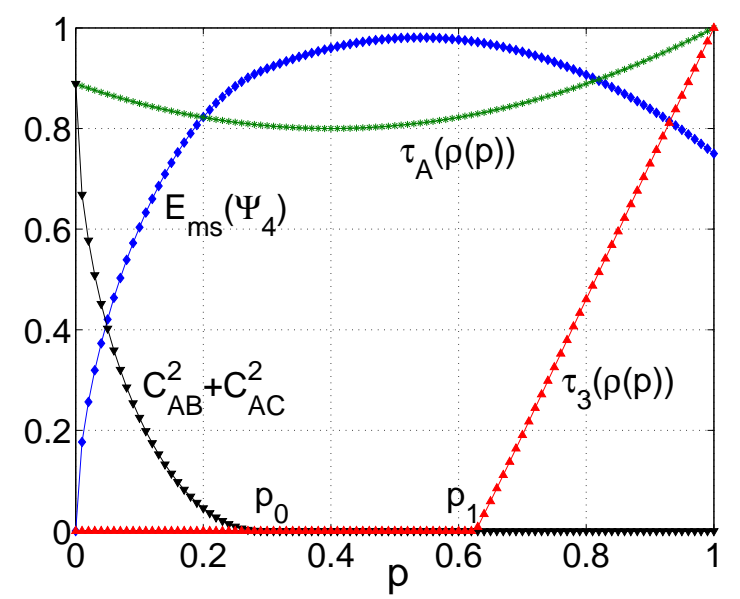

FIG. 1: (Color online) The average multipartite entanglement $E_{m s}$ (blue line) versus the decomposition parameter $p$ in the purified state $\left|\Psi_{4}\right\rangle$, in comparison with the concurrence (black line), the three-tangle (red line), and the one-tangle (green line) of the state $\rho(p)$ plotted in Ref. [15].

[20],

$$
E_{m s}\left(\Psi_{N}\right)=\frac{\sum_{k} \tau_{k\left(R_{k}\right)}-2 \sum_{i<j} C_{i j}^{2}}{N}
$$

which is used to characterize total multi-qubit entanglement, i.e., the sum of genuine three-, four-, ... , $\mathrm{N}$-qubit entanglements in the pure state. Here, the two-qubit entanglement in $\left|\Psi_{N}\right\rangle$ is quantified by the square of the concurrence which is defined as $C_{i j}=$ $\max \left[0,\left(\sqrt{\lambda_{1}}-\sqrt{\lambda_{2}}-\sqrt{\lambda_{3}}-\sqrt{\lambda_{4}}\right)\right]$ with the decreasing positive real numbers $\lambda_{i}$ being the eigenvalues of the matrix $\rho_{i j}\left(\sigma_{y} \otimes \sigma_{y}\right) \rho_{i j}^{*}\left(\sigma_{y} \otimes \sigma_{y}\right)[11]$. In a three-qubit pure state, $E_{m s}$ characterizes genuine tripartite entanglement and is just the three-tangle $\tau_{3}$. For the four-qubit case, $E_{m s}$ quantifies the sum of genuine three- and four-qubit entanglements. The result of much numerical analysis agrees with the conjecture that $E_{m s}$ is entanglement monotone [20]. Especially, for four-qubit cluster-class states, an analytical proof is given and a set of hierarchy entanglement measures is obtained 21, 22]. In a general $N$-qubit pure state, $E_{m s}$ is conjectured to be entanglement monotone. In Eq.(3), when the concurrences are zero, it is obvious that the property holds.

Entangled three-qubit mixed states-Lohmayer, et al. considered the following mixed state [15]

$$
\rho_{A B C}(p)=p|G H Z\rangle\langle G H Z|+(1-p)| W\rangle\langle W|,
$$

where the real parameter $p$ ranges in $[0,1]$, and the two orthogonal pure states have forms $|G H Z\rangle=(|000\rangle+$ $|111\rangle) / \sqrt{2}$ and $|W\rangle=(|001\rangle+|010\rangle+|100\rangle) / \sqrt{3}$, respectively. As shown in Fig.1, the quantum state is entangled $\left(\tau_{A} \neq 0\right)$ in the region $\left[p_{0}, p_{1}\right]$, but the entanglement is neither two-qubit entanglement nor genuine three-qubit entanglement (here $p_{0} \approx 0.2918$ and $p_{1} \approx 0.6269$ ).
With the reduction interpretation of mixed states, we consider a pure state $|\Psi\rangle$, which satisfies $\operatorname{tr}_{\mathcal{E}}(|\Psi\rangle\langle\Psi|)=$ $\rho_{A B C}(p)$ with $\mathcal{E}$ being environment system. Because $\rho_{A B C}(p)$ is a rank-2 quantum state, it is sufficient to consider an environment of dimension- 2 according to the purification theorem (a corollary of Schmidt decomposition) [23]. Therefore, the environment system $\mathcal{E}$ is equivalent to a qubit. For simplification, we consider the pure state

$$
\left|\Psi_{4}\right\rangle=\sqrt{1-p}|W\rangle_{A B C}|0\rangle_{D}+\sqrt{p}|G H Z\rangle_{A B C}|1\rangle_{D},
$$

where the environment is represented by qubit $D$. Any other purified state is equivalent to the $\left|\Psi_{4}\right\rangle$ plus a local unitary transformation $U_{D}$, which does not change the entanglement.

Since $\rho_{A B C}$ is a mixed state, the purified state $\left|\Psi_{4}\right\rangle$ is bipartite entangled in the partition $A B C \mid D$. We first analyze two-qubit entanglement in the pure state. Due to the permutation invariance of qubits $\mathrm{A}, \mathrm{B}$, and $\mathrm{C}$, there are only two independent two-qubit reduced density matrices, i.e., $\rho_{A B}$ and $\rho_{A D}$. Entanglement in subsystem $A B$ is $C_{A B}^{2}=\left(\max \left[0, \frac{2}{3}(1-p)-\sqrt{\frac{p}{3}(2+p)}\right]\right)^{2}[15]$. For the quantum state $\rho_{A D}$, we have $C_{A D}^{2}=0$. In threequbit reduced density matrices, only $\rho_{A B C}$ and $\rho_{A B D}$ are independent. An analytical formula of three-tangle for the quantum state $\rho_{A B C}(p)$ was given in Ref. [15]. However, the case for mixed state $\rho_{A B D}$ is different; it has the form

$$
\rho_{A B D}\left(\alpha_{p}, p\right)=\alpha_{p} \psi^{(1)}(p)+\left(1-\alpha_{p}\right) \psi^{(2)}(p),
$$

where $\psi^{(i)}=\left|\psi^{(i)}\right\rangle\left\langle\psi^{(i)}\right|$ is a projector with $\left|\psi^{(1)}(p)\right\rangle=$ $\sqrt{1-a}|000\rangle+\sqrt{a}|111\rangle$ and $\left|\psi^{(2)}(p)\right\rangle=\sqrt{b}|001\rangle+$ $\sqrt{(1-b) / 2}(|010\rangle+|100\rangle)$, and the coefficients are $\alpha_{p}=$ $(2+p) / 6, a=3 p /(2-p)$ and $b=3 p /(4-p)$, respectively. Eltschka, et al obtained an expression of three-tangle for this kind of mixed states [24]. We will show below that

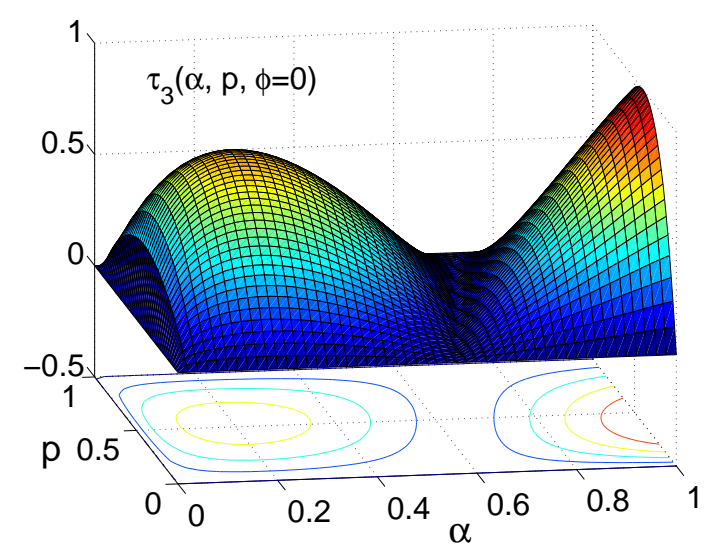

FIG. 2: (Color online) The pure state three-tangle $\tau_{3}\left(\Phi_{A B D}\right)$ versus the parameters $p$ and $\alpha$ for the relative phase $\phi=0$. 
this mixed state three-tangle is equal to zero. In general, the pure-state component of $\rho_{A B D}$ can be written as $|\Phi\rangle_{A B D}=\sqrt{\alpha}\left|\psi^{(1)}(p)\right\rangle-e^{i \phi} \sqrt{1-\alpha}\left|\psi^{(2)}(p)\right\rangle$, where real number $\alpha$ ranges in $[0,1]$ and relative phase $\phi$ in $[0,2 \pi]$. Its pure state three-tangle is

$$
\tau_{3}(\alpha, p, \phi)=4\left|f_{1}(\alpha, p)-e^{i 3 \phi} f_{2}(\alpha, p)\right|,
$$

where $f_{1}=6 \alpha p(1-p) /(2+p)^{2}$ and $f_{2}=24(p-$ $\left.p^{2}\right) /(4-p)\left[\alpha(1-\alpha)^{3} /\left(8+2 p-p^{2}\right)\right]^{1 / 2}$, respectively. When the relative phase $\phi=2 k \pi / 3$, given a value of parameter $p$, there always exists a nontrivial zero point $\alpha_{0}(p)=\left[1+(2 \sqrt[3]{2})^{-1}(6 /(2+p)-1)\right]^{-1}$ at which the pure state three-tangle is zero (in Fig.2, the $\tau_{3}\left(\Phi_{A B D}\right)$ is plotted as functions of the parameters $\alpha$ and $p$, where the relative phase $\phi=0$ is chosen). The cases for relative phases $\phi=2 \pi / 3$ and $\phi=4 \pi / 3$ are the same due to the phase factor $e^{3 i \phi}$ in Eq.(7). Therefore, the $\tau_{3}$ for mixed state $\rho_{A B D}\left(\alpha_{0}(p), p\right)$ is zero and its optimal decomposition is $\left\{\Phi_{k}(2 k \pi / 3)\right\}$ with probabilities $p_{k}=1 / 3$ for $k=1,2,3$. According to the convex characteristic manifold [25], we have $\tau_{3}\left(\rho_{A B D}(\alpha, p)\right)=0$ when $\alpha<\alpha_{0}(p)$, which is because the quantum state can be decomposed into the mix of $\rho_{A B D}\left(\alpha_{0}, p\right)$ and $\psi_{A B D}^{(2)}$ (a $W$-class state). In Eq. (6), the parameter $\alpha_{p}=(2+p) / 6$ ranges in $[1 / 3,1 / 2]$ which is less than the $\alpha_{0}(p) \in[0.5575,0.7159]$, therefore $\tau_{3}\left(\rho_{A B D}\right)=0$. In addition, we have $\tau_{3}\left(\rho_{A C D}\right)=\tau_{3}\left(\rho_{B C D}\right)=0$ in terms of the permutation invariance.

For the whole pure state $\left|\Psi_{4}\right\rangle$ in Eq. (5), we can derive the multipartite QCR entanglement

$$
\begin{aligned}
& E_{m s}^{I}\left(p \leq p_{0}\right)=\frac{3 p(2-3 p)}{4}+\frac{2(1-p) \sqrt{p(2-p)}}{\sqrt{3}} \\
& E_{m s}^{I I}\left(p>p_{0}\right)=\frac{8+(14-13 p) p}{12}
\end{aligned}
$$

which characterizes the genuine three- and four-qubit entanglements. As shown in Fig.1, we plot the variation of the measure along with parameter $p$. In the region $\left[0, p_{1}\right]$, due to all the three-qubit entanglement $\tau_{3}\left(\rho_{i j k}\right) \mathrm{s}$ being zero, the $E_{m s}$ quantifies only the genuine four-qubit entanglement $\tau_{4}$ which attains to the maximum 0.9808 at $p=7 / 13$. When $p_{1}<p \leq 1$, though the tripartite entanglement $\tau_{3}\left(\rho_{A B C}\right)$ is increasing, the multipartite entanglement $E_{m s}$ decreases with the parameter $p$. This is because the decrease of $\tau_{4}$ is stronger than the increase of $\tau_{3}$.

The mixed state one-tangle $\tau_{A}\left(\rho_{A B C}\right)$ is nonzero in the whole region (green line in Fig.1), which means the subsystems $A$ and $B C$ are always entangled. However, in the region $\left[p_{0}, p_{1}\right]$, the entanglement is neither twoqubit nor genuine three-qubit entanglement [15]. Our understanding lies in the fact that here the entanglement comes from a kind of genuine four-qubit $Q C R$ entanglement of the system $A B C$ and its environment $D$ since all the 2-, and 3-tangles are zero in the purified state $\left|\Psi_{4}\right\rangle$. The genuine four-qubit entanglement can reduce by a positive operator value measure (POVM) on qubit

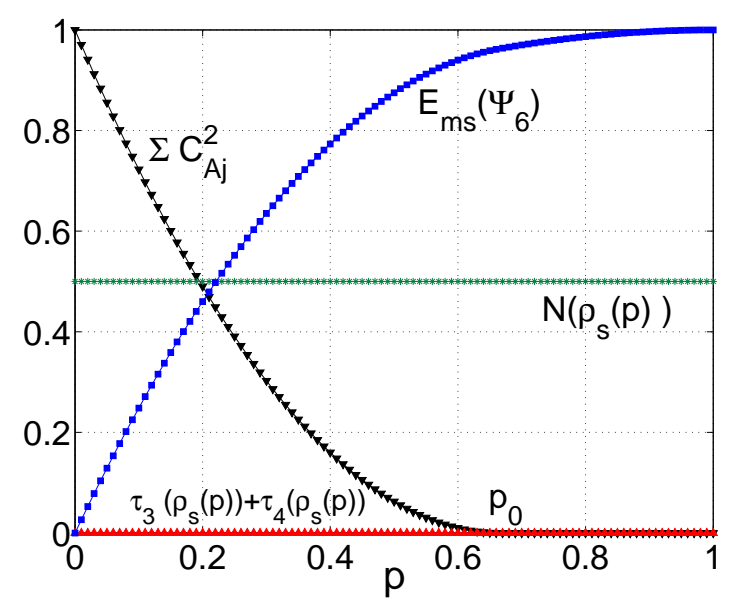

FIG. 3: (Color online) The average multipartite entanglement $E_{m s}\left(\Psi_{6}\right)$ (blue line), average negativity $\mathcal{N}\left(\rho_{S}(p)\right)$ (green line), concurrence sum $\sum C_{A j}^{2}$ (black line), and the sum of threeand four-tangle $\tau_{3}\left(\rho_{S}(p)\right)+\tau_{4}\left(\rho_{S}(p)\right)$ (red line) as a function of $p$.

$D$. The one-tangle $\tau_{A}\left(\rho_{A B C}\right)=\min \sum p_{i} \tau_{A}\left(\psi_{A B C}^{i}\right)$ characterizes the minimal average entanglement in the partition $A \mid B C$, for which its pure state component in the optimal decomposition has the form $\left|\psi\left(\varphi_{k}\right)\right\rangle=$ $\sqrt{p}|G H Z\rangle-e^{i \varphi_{k}} \sqrt{1-p}|W\rangle$. A two-component decomposition $\left\{\varphi_{1}=0, \varphi_{2}=\pi\right\}$ corresponds to a projection measure on environment $D$. In this case, the genuine four-qubit entanglement $\tau_{4}\left(\Psi_{4}\right)$ reduces to bipartite entanglement as measured by the one-tangle. The case for the decomposition $\left\{\varphi_{1}=0, \varphi_{2}=\frac{2 \pi}{3}, \varphi_{3}=\frac{4 \pi}{3}\right\}$ is similar and related to a three-outcome POVM.

Entangled four-qubit mixed states-The one-parameter Smolin state is

$$
\rho_{S}(p)=\frac{p}{4} \sum_{i=1}^{3} \psi_{A B}^{(i)} \otimes \psi_{C D}^{(i)}+\left(1-\frac{3 p}{4}\right) \psi_{A B}^{(4)} \otimes \psi_{C D}^{(4)},
$$

where $\psi^{(i)}=\left|\psi^{(i)}\right\rangle\left\langle\psi^{(i)}\right|$ is the projector on Bell basis $\left\{\left|\Phi^{ \pm}\right\rangle,\left|\Psi^{ \pm}\right\rangle\right\}[18]$. The quantum state has the symmetry of qubit permutations $A \leftrightarrow B, C \leftrightarrow D$, and $A B \leftrightarrow C D$. When the parameter $p=1, \rho_{S}$ is the original Smolin state [26, 27] which can maximally violate Bell inequalities and lead to secure quantum secret sharing. In the mixed state $\rho_{S}(p)$, there are only two independent two-qubit reduced density matrices, for which the entanglements are $C_{A B}^{2}=[\max (0,1-3 p / 2)]^{2}$ and $C_{A C}^{2}=0$, respectively. For the three-qubit reduced density matrix $\rho_{i j k}$, its pure state component can be written as $|\mu\rangle_{i j} \otimes|\nu\rangle_{k}$, so the three-tangle $\tau_{3}\left(\rho_{i j k}\right)=0$. The pure state component of $\rho_{S}(p)$ is the tensor product state of two Bell states. According to the formula $\tau_{4}(\rho)=\min \sum p_{i} \tau_{4}\left(\psi_{i}^{c}\right)$ [21], we can derive the fourtangle $\tau_{4}\left(\rho_{S}(p)\right)=0$. In Fig.3, we plot the concurrence, three- and four-tangles as a function of the parameter $p$. It is found that all entanglements are zero in the 
region $\left[p_{0}, 1\right]$ (where $\left.p_{0}=2 / 3\right)$. However, the negativity 17] $\mathcal{N}_{A}\left(\rho_{S}(p)\right)=\left(\left\|\rho_{S}^{T_{A}}(p)\right\|-1\right) / 2$ is nonzero in the region (the norm is defined as $\|\sigma\|=\operatorname{tr} \sqrt{\sigma \sigma^{\dagger}}$ ), which means that bipartite entanglement exists in the partition $A \mid B C D$ (due to the computational complexity, we do not choose the mixed-state one-tangle). The cases for partitions $B|A C D, C| A B D$ and $D \mid A B C$ are similar, and we plot the average negativity $\mathcal{N}\left(\rho_{S}(p)\right)=\sum \mathcal{N}_{k}\left(\rho_{S}(p)\right) / 4$ in Fig.3. In analogy to the three-qubit case, we show here that the negativity is only connected to a kind of genuine multipartite QCR entanglement between the system and its environment.

According to the purification theorem, it is sufficient to consider an environment $\mathcal{E}$ of dimension- 4 . Therefore, the $\mathcal{E}$ is equivalent to two qubits and the purified state of $\rho_{S}(p)$ can be written as

$$
\left|\Psi_{6}(p)\right\rangle=\sum_{i, j=0,1} \xi_{i j}\left|\psi^{(i j)}\right\rangle_{A B}\left|\psi^{(i j)}\right\rangle_{C D} \otimes|i j\rangle_{E F},
$$

where the $\left|\psi^{(i j)}\right\rangle$ is the Bell state, and the coefficients are $\xi_{00}=\xi_{01}=\xi_{10}=\sqrt{p / 4}$ and $\xi_{11}=\sqrt{1-3 p / 4}$. For this pure state, the multipartite QCR entanglement $E_{m s}\left(\Psi_{6}\right)$ characterizes the sum of genuine three, four-, five-, and six-qubit entanglements. After some derivation, we have $E_{m s}\left(p \leq p_{0}\right)=5 p(1-p) / 3$ and $E_{m s}\left(p>p_{0}\right)=\left(2+2 p-p^{2}\right) / 3$. In the quantum state $\left|\Psi_{6}\right\rangle$, the pure-state component of reduced density matrices $\rho_{i j k}$ and $\rho_{i j k l}$ can be written as a tensor product state. Therefore the mixed-state three- and four-tangles are zero in the purified state, and the $E_{m s}$ quantifies only genuine five- and six-qubit QCR-entanglement. After a POVM on the environment system $E F$, the entanglement $E_{m s}\left(\Psi_{6}\right)$ reduces to the bipartite entanglement in the one-parameter Smolin state as measured by the negativity $N\left(\rho_{S}(p)\right)$ in Fig.3 (blue line). It should be noted that the quantum state $\left|\Phi_{6}\right\rangle=U_{E F}\left|\Psi_{6}\right\rangle$ is also the purification of state $\rho_{S}(p)$, in which the unitary operation affects the concurrence $C_{E F}$ and the multipartite entanglement $E_{m s}$. However, it does not change our conclusion.

Discussion and conclusion- For the $N$-qubit case, there also exists the entangled mixed state in which the $k$-tangles entanglement are all vanished( here, $k=$ $2,3, \ldots, N)$. Because $\rho_{N}$ is a mixed state, the purified state $|\Psi\rangle_{N \mathcal{E}}$ is entangled in the partition $N \mid \mathcal{E}$. The entanglement $E_{N \mid \mathcal{E}}$ has a hierarchy structure, in which, after a POVM on environment $\mathcal{E}$, the genuine multiqubit QCR entanglements $\left\{E_{A_{1} A i \mathcal{E}}, E_{A_{1} A_{i} A_{j} \mathcal{E}}, \ldots, E_{A_{1} A_{2}, \ldots, A_{N} \mathcal{E}}\right\}$ re- duce to the bipartite entanglement measured by mixed state one-tangle $\tau_{A_{1}}$. As an example, we consider a quantum state given by

$$
\rho_{A_{1} A_{2} \ldots A_{N}}=\alpha\left|1^{\otimes N}\right\rangle\left\langle 1^{\otimes N}|+(1-\alpha)| W_{N}\right\rangle\left\langle W_{N}\right|,
$$

where $\left|W_{N}\right\rangle$ is an $N$-qubit $W$ state with the parameter chosen as $\alpha=1 /(N+1)$. The quantum state is invariant under qubit permutation, and there is only one independent two-qubit density matrix $\rho_{A_{i} A_{j}}$. After a simple derivation, one can obtain $C_{i j}=0$. Furthermore, one can deduce the higher tangles $\tau_{k}\left(\rho_{N}\right)=0$ for $k=3,4, \ldots, N$, which is because the quantum state can be written as the mix of a product state and a $W$ state. However, the mixed state one-tangle is $\tau_{A_{1}}\left(\rho_{N}\right)=4(N-1) /\left(N^{2}+N\right)$, which means that the mixed state $\rho_{A_{1} A_{2} \ldots A_{N}}$ is entangled. According to the purification theorem, its purified state has the form $\left|\Psi_{N+1}\right\rangle=\sqrt{\alpha}\left|1^{\otimes N+1}\right\rangle+\sqrt{1-\alpha}\left|W_{N}\right\rangle \otimes$ $|0\rangle$. After a POVM on the environment system $\rho_{A_{N+1}}$, the genuine multiqubit QCR entanglements between system and environment reduces to the one-tangle in the partition $A_{1} \mid A_{2} \ldots A_{N}$.

We have chosen the measure $E_{m s}$ to quantify the total genuine multi-qubit entanglement in a pure state. When the mixed state higher tangles $\tau_{k}(k>2)$ vanish, they are compatible with the $E_{m s}$. While we need to consider their compatibility whenever these tangles are nonzero, the analytical solutions for higher tangles of mixed state are still awaited, especially for the nonzero-tangle case.

In conclusion, with the help of the QCRs and a purification scenario, we have analyzed the one-tangle (or negativity) in a class of multi-qubit mixed states without multipartite tangles. It has been found that the entanglement in the purified state plays an important role. Especially, whenever the mixed state has no concurrence and higher tangles, its entanglement is just a kind of the genuine multipartite QCR-entanglement between the mixed state system and its environment.

Acknowledgments.- The authors would like to thank G.-P. He, M. Yang and Z.-Y. Xue for many helpful discussions. The work was supported by the RGC of Hong Kong under Grants No. HKU7051/06P, HKU7044/08P, and HKU-3/05C, the URC fund of HKU, and NSF China Grant No. 10429401. Y. K. B. was also supported by the fund of Hebei Normal University. M.Y.Y. was also supported by the Foundation for Universities in Fujian Province (Grant No. 2007F5041) and NSF-China Grant No. 60878059 .
[1] A. K. Ekert, Phys. Rev. Lett. 67, 661 (1991).

[2] C. H. Bennett and S. J. Wiesner, Phys. Rev. Lett. 69, 2881 (1992).

[3] C. H. Bennett et al, Phys. Rev. Lett. 70, 1895 (1993).

[4] C. H. Bennett and D. P. Divincenzo, Nature 404, 247 (2000).
[5] R. Raussendorf and H. J. Briegel, Phys. Rev. Lett. 86, 5188 (2001).

[6] S.-S. Li, G.-L. Long, F.-S. Bai, S.-L. Feng, and H.-Z. Zheng, Pro. Natl. Acad. Sci. USA, 98(21), 11847 (2001).

[7] M. B. Plenio and S. Virmani, Quantum Inf. Comput. 7, 1 (2007); R. Horodecki, et al, arXiv:quant-ph/0702225 2; 
L. Amico, et al, Rev. Mod. Phys. 80, 517 (2008).

[8] W. Dür, et al, Phys. Rev. Lett. 83, 3562 (1999); A. Acin, et al, ibid. 87, 040401 (2001); T. J. Osborne et al, ibid. 96, 220503 (2006); D. Yang, et al, ibid. 101, 140501 (2008).

[9] F. Verstraete, et al, Phys. Rev. A 68, 012103 (2003); C.S. Yu, et al, ibid. 71, 042331 (2005); A. Osterloh, et al, ibid. 72, 012337 (2005); J.-M. Cai, et al, ibid. 74, 042338 (2006); D. L. Zhou, et al, ibid. 74, 052110 (2006); Y.-C. $\mathrm{Ou}$, et al, ibid. 75, 062308 (2007).

[10] A. Uhlmann, Open Syst. Inf. Dyn. 5, 209 (1998); A. Uhlmann, Phys. Rev. A 62, 032307 (2000).

[11] S. Hill and W. K. Wootters, Phys. Rev. Lett. 78, 5022 (1997); W. K. Wootters, Phys. Rev. Lett. 80, 2245 (1998).

[12] V. Coffman, J. Kundu, and W. K. Wootters, Phys. Rev. A 61, 052306 (2000).

[13] E. Santos and M. Ferrero, Phys. Rev. A 62, 024101 (2000).

[14] W. Dür et al, Phys. Rev. A 62, 062314 (2000).

[15] R. Lohmayer et al, Phys. Rev. Lett. 97, 260502 (2006).
[16] O. Cohen, Phys. Rev. Lett. 80, 2493 (1998).

[17] G. Vidal and R. F. Werner, Phys. Rev. A 65, 032314 (2002).

[18] J. A. Smolin, Phys. Rev. A 63, 032306 (2001).

[19] M. Jakob and J. A. Bergou, arXiv:quant-ph/0302075, T. E. Tessier, Found. Phys. Lett. 18, 107 (2005); X. Peng et al, Phys. Rev. A 72, 052109 (2005).

[20] Y.-K. Bai, et al, Phys. Rev. A 76, 022336 (2007).

[21] Y.-K. Bai, and Z. D. Wang, Phys. Rev. A 77, 032313 (2008).

[22] X.-J. Ren, et al, Phys. Rev. A 78, 012343 (2008).

[23] A. Peres, Quantum Theory: Concepts and Methods, (Kluwer, Dordrecht, 1993) p123.

[24] C. Eltschka, et al, arXiv:0711.4477 [quant-ph].

[25] A. Osterloh, et al, Phys. Rev. A 77, 032310 (2008).

[26] R. Augusiak and P. Horodecki, Phys. Rev. A 74, 010305(R) (2006); Y. Yu, Phys. Rev. A 75, 066301 (2007); G.-P. He, et al, J. Phys. A 41, 415304 (2008).

[27] M. Hillery et al, Phys. Rev. A 59, 1829 (1999). 